\section{HOW PARENTS TO TECHNOLOGY DEPENDENT CHILDREN EXPERIENCE THE TRANSITION FROM A HOSPITAL STAY TO PROFESSIONAL HOME CARE NURSING}

\author{
M.S. Lind \\ Childrens Intensive Care, Aarhus University \\ Hospital, Skejby, Aarhus, Denmark
}

The object of the study: This study investigates how parents to a young or newborn Technology Dependent Child (TDC) experience the transition from a long term hospital stay with their child to professional home care nursing on a daily basis.

Method: A qualitative semistructured interview method was used to interview the parents of five children who had been discharged from the hospital with a (TDC) within two years. The five interviews were transcribed and were subsequently analysed and interpreted based on Paul Ricoeur's interpretive framework.

Findings: The parents asked for more support from the professional and new knowledge as parents in their new life situation. They felt unsure how to relate to the home care professional with trust in the beginning. Data analysis identified the following themes: Finding support, finding trust and finding power, knowledge and strength to cope with the new situation and to cope with having professionals in their home on a daily basis.

Conclusion: In conclusion, this transition is not unproblematic for the parents of a (TDC). The professionals involved in the transition need to pay more attention to the parent needs and knowledge before the transition to the home. A better understanding of the parents individually needs according to their (TDC's) care and their family life should be more in focus by the professionals working in the home. The professionals need a closer collaboration with each other, to support the parents and to make them feel safe in their new situation.

115

PEDIATRIC RAPID RESPONS TEAM

\section{T. de Raad}

PICU, UMC Utrecht, Utrecht, The Netherlands

Background and aims: Cardiac arrest in hospitalized children has a poor prognosis. Only approximately $25 \%$ of the arrested children survive. Evidence shows that death form unexpected but foreseeable cardiac arrest is an adverse event that may be preventable with timely intervention. In some hospitals there are specialized teams of doctors and nurses who aim to treat critically ill patients before cardiac of respiratory arrest occurs. Rapid response teams were first developed within the adult medical community, and appear to be successful.

Methods: The goal is to search for evidence of the effectiveness of a pediatric rapid response team. The study is designed as a literature study to find the highest level of evidence in scientific literature for the effect of a pediatric RRT in decreasing cardiac arrest rates and decreasing mortality.

Results: Recent studies have encouraging results, decreasing cardiac and respiratory arrest and decreased mortality. However the results according the mortality rate are not conclusive and relatively few data regarding pediatric experience with RRTs have been published.

Conclusion: The data on RRT's in pediatric hospitals are increasing and thus far suggest benefit. The challenge is to collect rigorous and comparable data, whether beneficial effects are demonstrated or not.

\section{6}

\section{MEASURING OF INTRA-ABDOMINAL PRESSURE IN CRITICALLY ILL CHILDREN BY NURSES}

M.M. van de Polder, S. Plug, C. Buth, N. van Beelen, R.J. Houmes, E. Ista

Pediatric Intensive Care Unit, Erasmus MC Sophia Children's Hospital, Rotterdam, The Netherlands

Objective of the study: Delayed diagnosis of increased intra-abdominal pressure in PICU patients increases morbidity and mortality rates. In 2007 our PICU implemented a protocol for indirect intra-abdominal pressure (IAP) measurements by an indwelling bladder catheter using a pressure transducer. One of the indications to start IAP measurement is an increased abdominal distension. The aim of this study was to evaluate the effects of this protocol, including the nurses' compliance.

Methods: This retrospective study included patients admitted between 2007-2009. Children who underwent IAP measurements and/or abdominal 